\title{
Daily solar radiation forecasting using historical data and examining three methods
}

\author{
Mehryar Parsi \\ Department of engineering, University Putra Malaysia
}

\begin{abstract}
In any renewable energy system, knowing about availability of resources is one of the main concern for scientists. In another words, challenge is when these resources are needed to be predicted. Forecasting solar radiation is one of the most noticeable unsettled case. By having a thought of forecasting solar radiation would be easier comparing to other renewable resources, because it is not related to wind speed or weather temperature. However, after this experiment it become more clear that, the amount of receiving radiation is proportional to weather alternative conditions such as movement of clouds, haze and even the density of them. In this paper, three different approaches were examined by using historical data to forecast solar radiation day by day. The three approaches are, exponential smoothing, seasonal forecast and feed forward neural network using MatLab. An important finding based on this experiment is that, forecasting radiation by using historical data is not fitting. Meaning to say that, by numbers alone, result of forecasting is getting close to random rather than being more realistic, because receiving radiation is affected by other aspects. For improving the prediction, a method is suggested in future studies.
\end{abstract}

Keywords: Daily, solar radiation, forecasting, historical data, exponential smoothing, seasonal, neural network, feed forward.

\section{Introduction}

Filling up electronic demands using renewable energy is getting more interesting every day, not just only it is free to get, above of that because it is pollution free. Renewable energy sources as photovoltaic (PV), wind, bio-diesel generator are now utilized in integrated generation systems at sites that have a large potential of either solar, wind or both. At present, solar photovoltaic and wind systems have been promoted around the globe on a comparatively larger scale [1-6]. As it mentioned, these energies are free but doesn't mean that they are always available. For instants, solar photovoltaic energy system cannot be useful at night and also cannot be satisfied during cloudy days. It goes the same way for wind system, it cannot be efficient even during windy days, because amount of wind speed is required to get into that level, which makes the prediction harder for calculations. Even though if winds speed is satisfied doesn't mean that it will be constant even for an hour. Therefore, for saving energy to meet demands few of these system need to work together so they may cover each other up if it is possible for having a better reliability.

Comparing to other efficient renewable energy system, solar is the most common cause, it is possible to use it daily, solar conversion, and renewable energy applications, particularly for the sizing of stand-alone photovoltaic (PV) systems [1]. Solar energy or radiation is not very easy to get as well, but comparing to others is more convenient, as it is known, in air there are a lot of particles which directly will effect on the solar radiation frequency that will effect on absorbing solar energy by PV's a good example would be clouds and haze. Complete sun radiation is the most essential alternative in forecasting of renewable energy systems, especially in designing PV power systems. Knowing a perfect sizing of solar system [2-4] (PV) is very important, by over-sizing the system, it doesn't mean that result will be better, because, by not considering prize and costs, by having a larger area, chances of having shaded area on PVs will increase which reduces efficiency of PVs performance quite a lot. In other hand, by having smaller size or in other word, under-sizing the system, reduces the power supply reliability [1]. That's why, accurate forecasting of radiation is one of the challenges for scientists, not just only to predict, for designing as well. For time being, there are many approaches for forecasting irradiance [5-21]. It has grown from mathematical formula and combination of them up to analyzing satellite images by using artificial intelligence. Yet, results were surprising not as accurate as it was expected [7]. In this review paper, three most common used approaches for forecasting irradiation [7-21] were examined, which are exponential smoothing, seasonal method and neural network. For all these three same historical database were used and at the end comparison between them were designed.

\section{Forecasting}

For having a more clear image and comprehend better of availability of solar radiation, and to give data for future planning, number of maps and data bases have been delivered by method for estimations and displaying. The most common models provide data for several climate parameters including solar irradiance. 
The quality of the numerical weather prediction (NWP) models has improved considerably over time and consequently the model output is not homogenous [2]. In order to produce more suitable with more accuracy data sets for planning and forecasting are past weather record can be analyzed in more detail it can be done by using devices that keep record of data, a good example would be satellites [4].

Forecasting techniques can be divided in to two main general class: based on numbers which is called quantitative and qualitative or subjective. The strategies in the quantitative class incorporate numerical models, for example, moving average, straight-line projection and exponential smoothing. In the subjective classification subjective or intuitive models such as jury or executive opinion, sales force composite, and customer expectations is the strategy [20].

Besides the two categories those were mentioned, there are other factors that will effect on forecasting such as time or duration which are called as time-series, causal, and judgmental [8]. A time-series model basically depends on the pervious recorded data to do the calculations for forecasting [11]. The concept of a causal model is that, for specific expected result which is directly affected by other predictable factor. These techniques include regression models. Judgmental techniques are basically lies on probability and calculations so that all the time it might not going to predict the result with same value for similar situations [8].

\section{Methodologies}

Exponential forecasting: based on the duration and amount of data, different formula need to be used, but generally for this paper, forecasting formulas such as seasonal forecasting (mathematical) and exponential smoothing were selected and applied by Excel. Besides, ANN was used as well to get more result and at the end, comparison between them is one of the way to show which forecasting is more suitable for this and as long as the errors are less the impotency of forecasting for renewable energy will be more noticeable.For forecasting the first approach was exponential smoothing, which is suggested for time series forecasting, that weather can be included as well.

$\mathrm{Ft}+1=\alpha \mathrm{Dt}+(1-\alpha) \mathrm{Ft}$

Where;

$\mathrm{Ft}+1=$ the forecast for the next period.

$\mathrm{Dt}=$ actual demand in the present period.

$\mathrm{Ft}=$ the previously determined forecast for the present period.

$\alpha=$ a weighting factor referred to as the smoothing constant.

For doing this method first the data are needed to be arranged based one the seasonality, after that, need to check whether there is co-relation between data so that forecasting can be applied. This can be done by taking the average of few years averages that are included the same set of data. As it mentioned before checking a ratio would be the next step. Of course, when talking about seasonal, day by day wouldn't be the option, even month to month comparison wouldn't show a suitable result, but still for this paper month by month and 6 months we calculated. The calculation of seasonal data for each selected month need to be started with the first averaging all the ratios for that particular season [7-8].

Seasonal approach: Second approach was seasonal forecasting, as long as the weather in would be almost the same as pervious years so using seasonal forecasting can be suitable. For doing this method Excel is highly recommended. For doing this approach few steps required to be calculated. (a) To sum up all data for a period for instance all data for six month, meanwhile average of a mentioned period needs to be computed (b) then the average of all these sums depending on how many periods are being looked for. For this case data was available for four continues years so the total divide by four to take the average of them and then divide this value with number of days depends on the mention period. For example, six month would be six time thirty days which is equal to hundred and eighty. (c) Looking at particular day and finding how much is median of that day for each year. (d) times step $\mathrm{c}$ value with the last part of $\mathrm{b}$ which leads to the forecast of each day of future period [10].

As it is known that weather conditions are not stable, so expectation of accuracy will be reduced. For having more accurate climate status, having more data for several particular period is suggested. For example for raining season in any country, having solar radiation for few years back such as five or eight years will give better result comparing to have two or three years. The thing that is needed to be mentioned here is that, seasonal climates will have the same pattern in comparison with months or even weeks. For getting more results, seasonal forecasting is applied for each months beside the seasonal period. But the formula and everything is same just the period has been changed. In other words, the duration of few months reduced to a month.

Neural network: In this paper, ANN in terms of feed forward method has been tried based on the papers regarding to using neural network for forecasting [14]. Which means for specific periods that is required to be forecast, historical data observation for predicting that specific duration is needed.The third and last method was neural network for forecasting to make the selection of which method easier based on the accuracy and comparison of errors. But how does ANN will help on this forecasting. It will be explained on figure 1. 


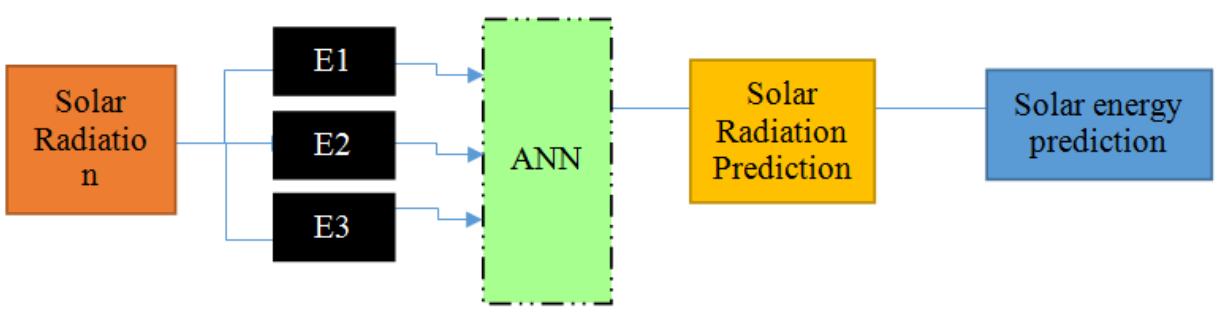

Figure 1. Overall view of neural network.

\section{Discussion}

The main aim is to create and train a network that can predict the solar radiation. For a specific location and for specific day or week which is going to be our output and the historical data for that specific location and duration would be the input for our artificial neural network (4 years data is provided for this paper). In this paper, solar radiation was focused because the main purpose of this paper was to examine how forecasting can be effective, since 2010 till 2014. So following up with this data was selected. The data was recorded by analyzing the graphs which one sample is available in table 1.

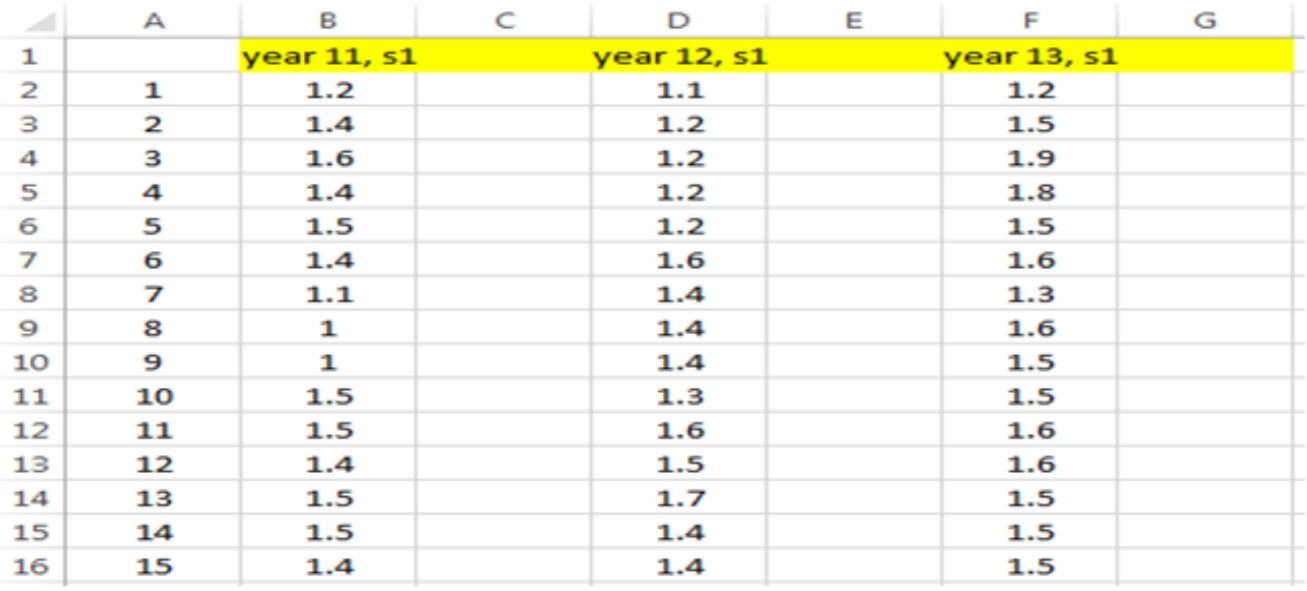

Table 1. Dataset from graph

At first it was exponential smoothing method. A excel file was made for 365 days of each year and it was plotted in MATLAB as data base in workplace. Started from January 2010 till January 2014. In front of each day solar radiation records of 3 years were written. In overall, out of 365 days $90 \%$ were selected for training and 5\% were selected for validation and the rest 5\% was for testing.
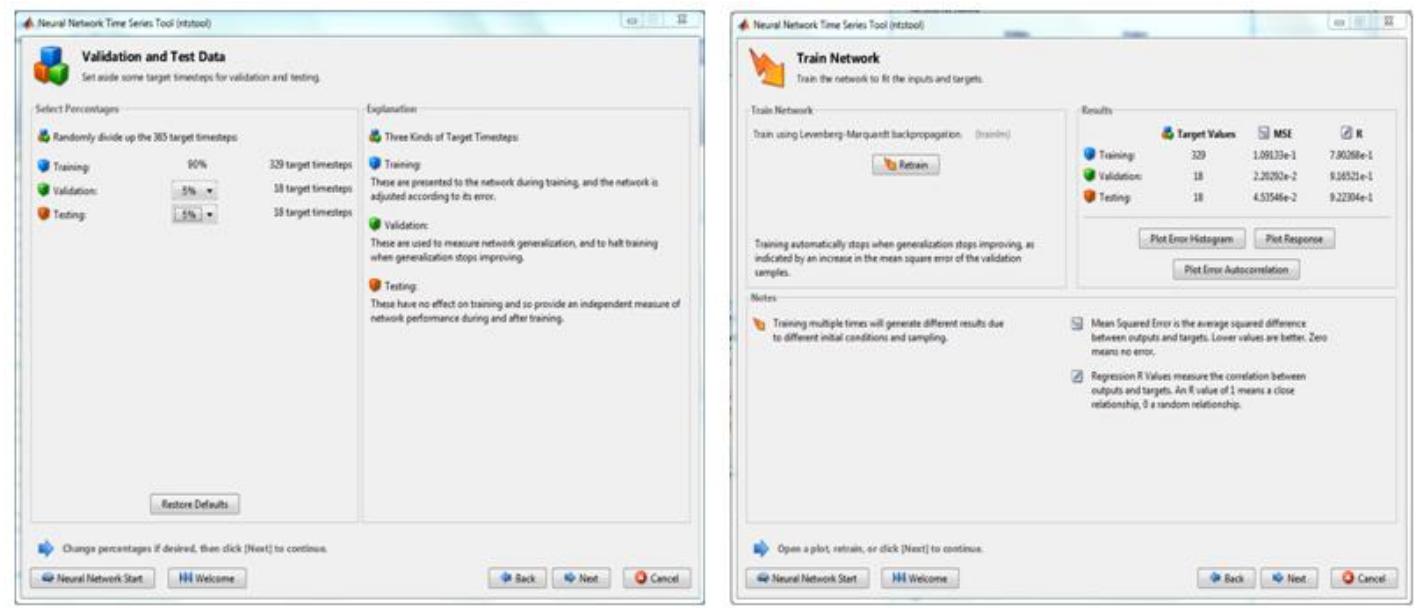

Figure 2. Training and checking R and MSE after training. 
Neural network is famous because it can take many data sets like 365 which is for one year and even more years. But in this paper input data set was just a year and expecting output also was 365 days. The output data was compared with real solar radiation which was available for each year. Next step would be checking the $\mathrm{R}$ and MSE, as much as MSE is closer to zero the result would be more suitable and based one readings on papers, as long as $\mathrm{R}$ is near or above 0.9 , the data sets are suitable.

By increasing hidden layers, MSE will be reduced, in this paper by adding more than 10, MSE was not changed noticeably. Besides, by increasing number of samples which are solar radiation of each year, MSE also will reduce, for doing so, solar radiation for 3 years were selected for input. As it is shown in figure 3, the MSE is almost near to zero, and the validation and training $\mathrm{R}$ is 0.9 which meets the requires. In next figure, the combination of training, validation and testing is shown.

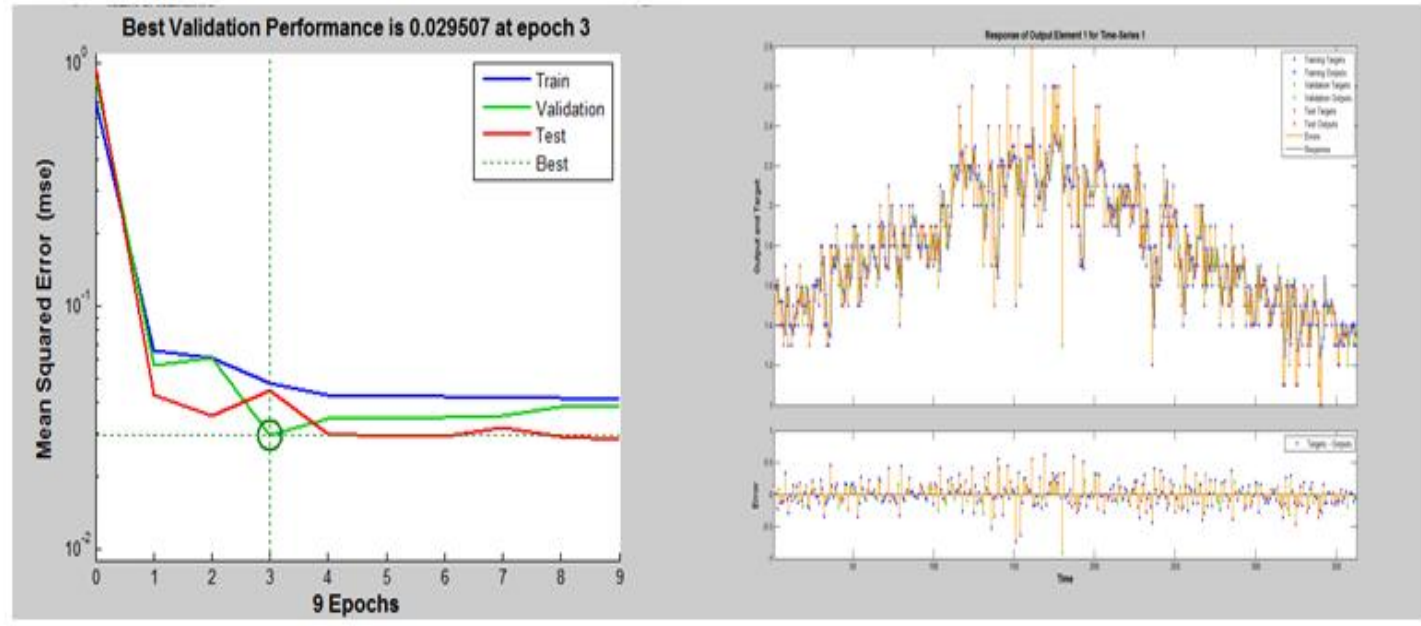

Figure 3. Validation, testing and errors.

After this the output data was compared with actual reading data from each year and the error was higher than what was expected. The average error for 2012, 2013 and 2014 was, 12\%, 13\% and 15\% respectively. The differences between actual and forecasted values by Neural Network is shown in graph below (figure 4).

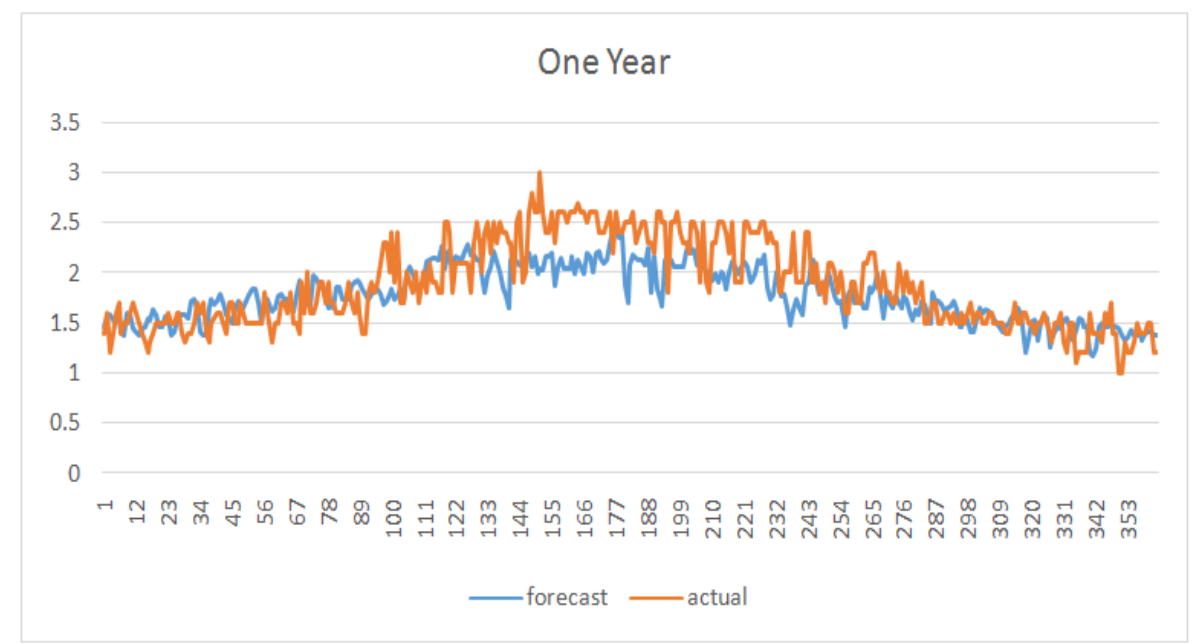

Figure 4. Neural network forecasting vs actual value.

Next step was seasonal forecasting. In other words, for specific period of time that is required to be forecasted, historical data for that specific period is required as it mentioned. For first six months of year 2014 , the average error was 20 percent and for other half the average error was 15 percent. By just looking at figure 5 , it is possible to see that almost 70 percent of forecasting and actual values are following same path and slop. The rest actually is the error which were expected when calculation was done. Based on this graph as long as the blue lane is under the actual value, we can say that the process based on the forecasting is safe. In other words, if a system needs to make decision making, as long as blue line is under actual value line, it won't be any problem. 


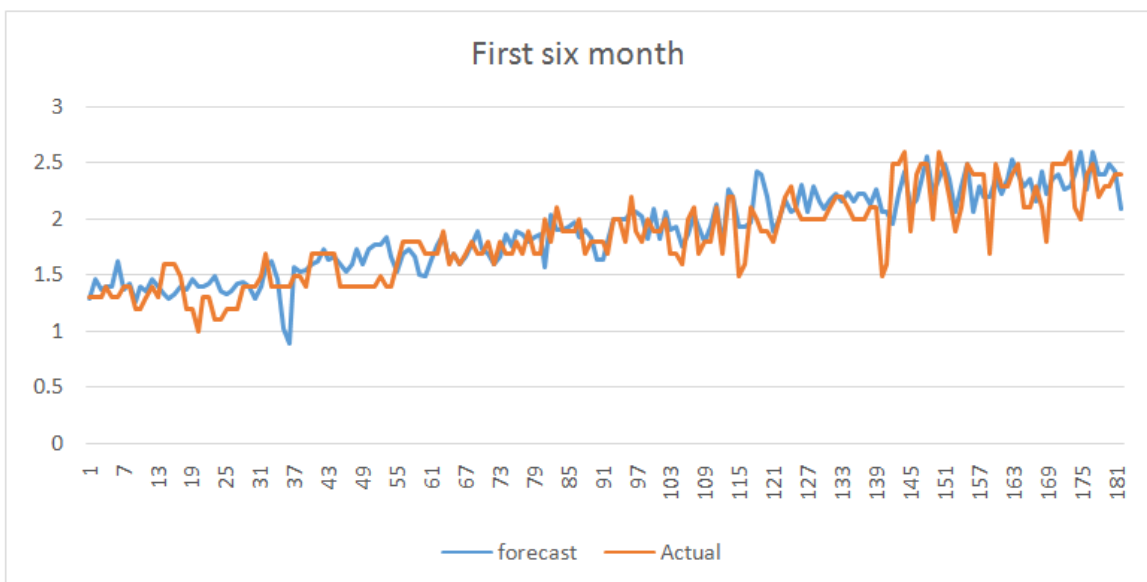

Figure 5. Seasonal forecasting for first six months vs actual value for 2014.

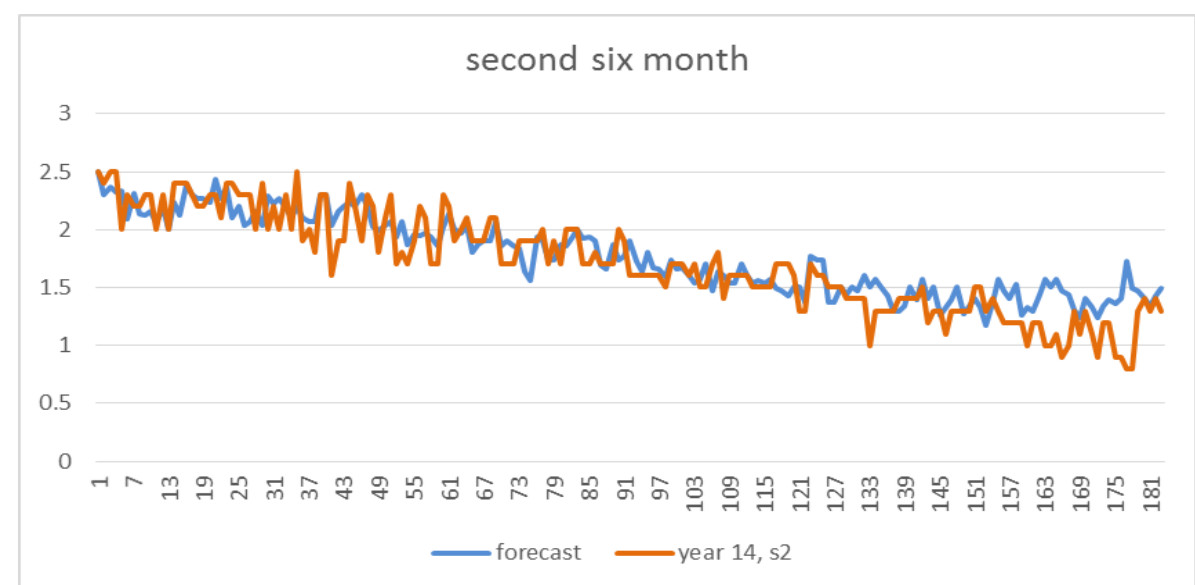

Figure 6. Seasonal forecasting of second six month vs actual value for 2014.

So basically, for doing exponential smoothing, every day will be counted for next day. By looking at the graph, it is possible to see the pattern will go up and down, logically by calculating a day or even few day before while the graph starts to increase or decrease, a suitable result won't appear much and the errors between actual and forecast would increase in these cases. However, these patterns are following the same path. So for this paper, forecasting was essential, few methods were selected to get more satisfying result. 365 days of a year was the input for this method, and the answer was compared with the next year solar radiation so for this method there is a value that is required to be selected by the person who is doing the calculations and this value need to be between 0 to 1 . For this paper all possible numbers from 0 to 1 were applied and the results was interestingly better than other methods. In next two figure the maximum average error for year 2014 was belong to Trial one (0.1) and the rest which means 0.2 to 0.9 with average of $9 \%$ and $8 \%$ respectfully.

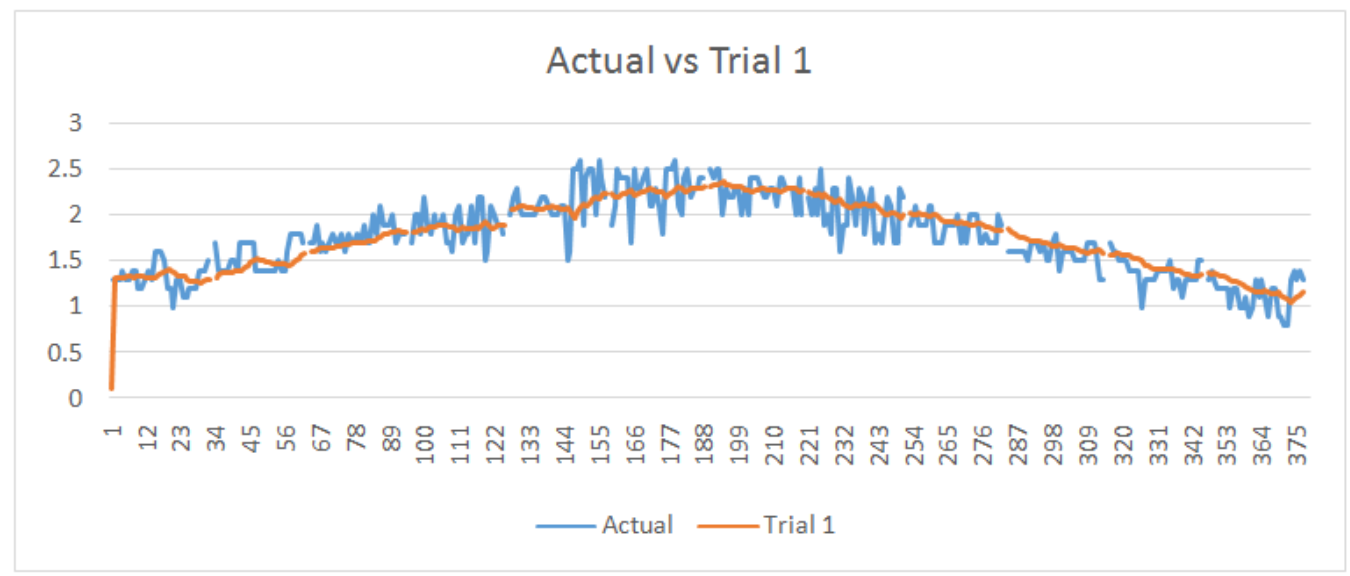

Figure 7. Exponential Smoothing. 
What can be seen from this graph is that the forecast and actual are not even close to each other comparing to Neural Network and seasonal. But the advantage of this method is that, it is not required to be exact as long as the forecasting is in the middle. For better understanding, for few days the actual value might be more than what has been predicated and in other hand the next few days the actual value might be less than what has been predicted.

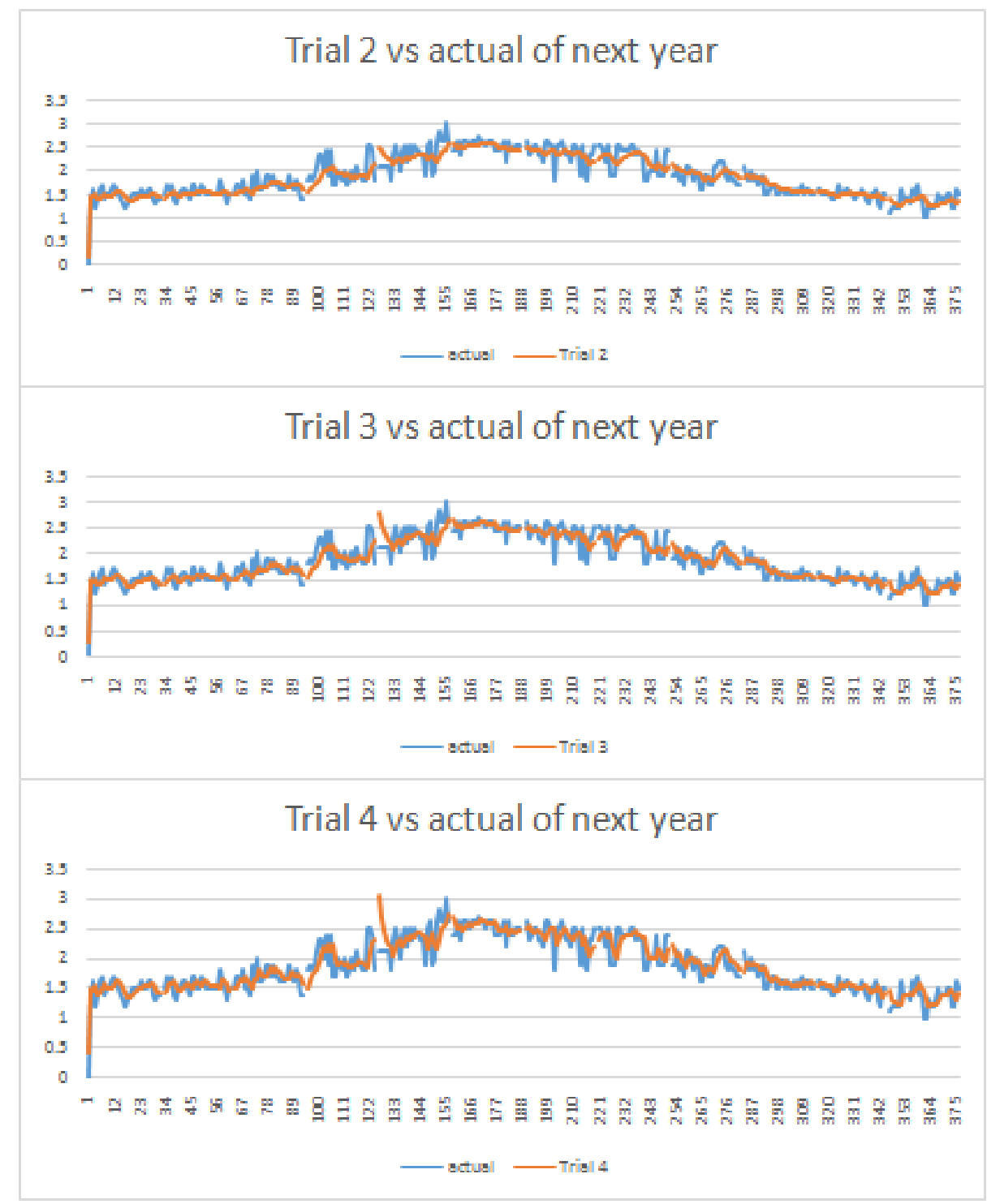

Figure 8. Comparing trial 2, 3 and 4 versus actual data.

For these captured charts, in fig 8, average errors were 12\%, $13 \%$ and $13 \%$ respectfully. For the rest of the Trials (trial5 - trial9) these charts are more or less the same and errors are; $13 \%, 13 \%, 14 \%, 14 \%$ and $14 \%$.

\section{Conclusion}

In this paper few forecasting method was used to get more accurate data comparing to one method. The methods are, exponential smoothing, seasonal forecasting and artificial neural network were used. At the end exponential smoothing had the less average errors comparing with the rest methods. In addition, the differences between each method's errors wasn't really high to make the decision of which method is the most suitable. For forecasting, the dataset was provided from a website that the dataset from website. These dataset was collected on ground surface condition. In other words, no angle between the sensor plane (panel) and ground surface. So based on this situation, it brings the opportunely to compare the best performance and the worst performance of designed solar panels. 
For further improvement, this paper dataset was provided by eye reading based on the graph. It is possible to have human error which will effect directly on accuracy of forecasting, because forecasting using mathematical way will definitely shows some errors and now even the accuracy of dataset is less as well so the errors of forecasting will increase even more. This paper can be applicable if it was possible to get dataset online. Meaning, if dataset could be updated, so it would help to forecasting to have more data, based on some readings, it is possible to program excel to update itself if there is a website that dataset is provided and it gets upgraded. Furthermore, regarding to use neural network, numbers and datasets are not really reliable based on the experiencing while comparing to exponential smoothing for weather forecasting. This artificial neural network can be used for more complicated forecasting by using real or live images from satellites of clouds and not by mathematical numbers only. Surely, by knowing the weather condition in past it would be a good reference but not the main part of forecasting calculations. For example, by seeing the speed and how clouds are moving from satellite image, neural network can guess what would be the next, so after this, it is possible to look for similar condition in past and get an idea of what to predict and expect. Still, need to keep in mind that there are other alternatives will affect the forecasting while using satellite images such as the density of clouds or haze. By training neural network to differentiate between clouds would reduce the errors even more.

\section{References}

[1] Nehrir, M.H. et al: A review of hybrid renewable/alternative energy systems for electric power generation: configuration, control and applications, IEEE Transactions on Sustainable Energy, Vol. 2, No. 4, 2011, pp. 392-403.

[2] JoakimWidén, NicoleCarpman, ValeriaCastellucci, DavidLingfors, JonOlauson, FloreRemouit, MikaelBergkvist, MårtenGrabbe, RafaelWaters; Variability assessment and forecasting of renewables: A review for solar, wind, wave and tidal resources, 2015, 356375.

[3] D. Connolly, H. Lund, B.V. Mathiesen, M. Leahy; 'The first step towards a 100\% renewable energy-system for Ireland',Volume 88, Issue 2, 2011, 502-507.

[4] TurkeyA. Tascikaraoglu, A.R. Boynuegri, M. Uzunoglu; 'A demand side management strategy based on forecasting of residential renewable sources: A smart home system in Turkey', Volume 80, 2014, 309-320.

[5] A. Ahmad, T.N. Anderson, T.T. Lie;Hourly global solar irradiation forecasting for New Zealand,Volume 122, 2015, 1398-1408.

[6] Xiaochen Wang, Peng Guoc, XiaobinHuanga; A Review of Wind Power Forecasting Models, Volume 12, $2011,770-778$.

[7] Zibo Dong, Dazhi Yang, Thomas Reindl, Wilfred M. Walsh; Short-term solar irradiance forecasting using exponential smoothing state space model, Volume 55,2013, 1104-1113.

[8] P. Y. Lim and C. V. Nayar; Solar Irradiance and Load Demand Forecasting based on Single Exponential Smoothing Method, Vol. 4, No. 4, 2012.

[9] EmreAkarslan, FatihOnurHocaoglu; A novel adaptive approach for hourly solar radiation forecasting, Volume 87, 2016, 628 - 633.

[10] R. Urraca, J. Antonanzas, M. Alia-Martinez, F.J. Martinez-de-Pison, F. Antonanzas-Torres; Smart baseline models for solar irradiation forecasting,Volume 108, 2016, 539-548.

[11] Yamin Wang, Lei Wu; On practical challenges of decomposition-based hybrid forecasting algorithms for wind speed and solar irradiation, Volume 112, 2016, $208-220$.

[12] Alexandros Agapitos, Michael O’Neill, and Anthony Brabazon; Evolving Seasonal Forecasting Models with Genetic Programming in the Context of Pricing Weather-Derivatives, 2012, pp 135-144.

[13] RahatHossaina, AmanullahMaung Than Ooa, A B M Shawkat Alia; Historical Weather Data Supported Hybrid Renewable Energy Forecasting using Artificial Neural Network (ANN), Volume 14,2012, 1035-1040

[14] Kalogirou S., Michaelides S., Tymvios F.: Prediction of maximum solar radiation using artificial neural networks, Volume 74,2011, $1205-1212$.

[15] K.Kadirgamaa, A.K.Amirruddina, R.A.Bakara; Estimation of Solar Radiation By Artificial Networks: East Coast Malaysia,Volume 52, 2014, 383-388.

[16] Paras, Sanjay Mathur, Avinash Kumar, and Mahesh Chandra; A Feature Based Neural Network Model for Weather Forecasting, 2008.

[17] Vishal Sharma, Dazhi Yang, Wilfred Walsh, Thomas Reindl; Short term solar irradiance forecasting using a mixed wavelet neural network, Volume 90, 2016, 481 - 492.

[18] Gaurav J. Sawale, Dr. Sunil R. Gupta; Use of Artificial Neural Network in Data Mining For Weather Forecasting, Vol. 6, No.2, 2013.

[19] James W. Taylor and Roberto Buizza; Neural Network Load Forecasting With Weather Ensemble Predictions, VOL. 17, NO. 3, 2002.

[20] M. C. Valverdea, Ernesto Araujob, H. Campos Velhoea, Neural network and fuzzy logic statistical downscaling of atmospheric circulation-type specific weather pattern for rainfall forecasting, Volume 22 2014, 681-694.

[21] Amit Tiwari, AdarshDhar Dubey, and Devesh Patel; Comparative Study Of Short Term Load Forecasting Using Multilayer Feed Forward Neural Network With Back Propagation Learning And Radial Basis Functional Neural Network, 2015.

[22] The International System of Units (SI). PDF. 